\title{
Essential Oil Composition and Antimicrobial Activities of Lavandula pubescens, Marrubium vulgare and Meriandra bengalensis from Yemen
}

\author{
Yani $\mathbf{A A}^{1}$, Eldahshan $\mathbf{O A}^{2,3 *}$, Hassan $\mathrm{SA}^{4}$, Elwan $\mathrm{ZA}^{4}$ and Ibrahim $\mathrm{HM}^{\mathbf{1}}$ \\ ${ }^{1}$ Biology Department, Botany Section, Faculty of Science, Sanaa University, Yemen \\ ${ }^{2}$ Pharmacognosy Department, Faculty of Pharmacy, Ain Shams University, Egypt \\ ${ }^{3}$ Center for Drug Discovery Research and Development, Ain Shams University, Egypt \\ ${ }^{4}$ Botany Department, Faculty of Science, Ain Shams University Cairo, Egypt \\ *Corresponding Author: Omayma A Eldahshan, Faculty of Pharmacy, Department \\ of Pharmacognosy, Ain Shams University, Cairo, Egypt.
}

Received: August 02, 2021

Published: September 18, 2021

(C) All rights are reserved by Eldahshan $\mathbf{0 A}$, et al.

\begin{abstract}
In Yemen, family Lamiaceae is one of the most important families due to its use in folk medicine and for the commercial production of essential oils. In this study, three Lamiaceae plants; Lavandula pubescens Decne, Marrubium vulgare L. and Meriandra bengalensis (Roxb.) Benth, were chemically investigated by GC/MS as well as their antimicrobial activity was assessed using agar-well diffusion method. The major compounds in Lavandula pubescens oil were carvacrol (54.98\%), thymol methyl ether (12.15\%) and Z-caryophyllene (4.47\%), while those of Marrubium vulgare were Z-caryophyllene (10.95\%), octadecanol (10.44\%) and $\alpha$-bisabolene (9.72\%). Meriandra bengalensis contained camphor (64.87\%) as a major compound with 1,8 cineole (12.16\%) and camphene (11.96\%). The strongest antimicrobial activity was observed in Lavandula pubescens against Klebsielle pneumoniae (1.95 $\mu \mathrm{L} / \mathrm{mL}) \mathrm{which}$ is higher than that of gentamycin, Enterobacter cloacae $(0.98 \mu \mathrm{L} / \mathrm{mL})$, and Bacillis subtilis $(0.49 \mu \mathrm{L} / \mathrm{mL})$ that are similar in activity to that of standards (gentamycin and ampicillin). Oils of Marrubium vulgare L. and Meriandra bengalensis exhibited a range of activities between weak and none. Our result concludes that Lavandula pubescens possess pronounced antimicrobial activity against Klebsielle pneumoniae, Enterobacter cloacae and Bacillis subtilis giving it a highly medicinal impact value to be further studied in vivo against different types of microbial infectious diseases
\end{abstract}

Keywords: Lamiaceae; Volatile Oil; Antimicrobial Activity

\section{Introduction}

Lamiaceae, formerly called Labiatae, the mint family of flowering plants, with 252 genera and 6700 taxa, the largest family of the order Lamiales. Lamiaceae is distributed nearly worldwide, and their species are cultivated for their fragrant leaves and attractive flowers. The family is of high importance for flavor, fragrance, or medicinal activities $[1,2]$.

In Yemen, Lamiaceae family is represented by 23 genus with 23 species are endemic to Yemen. Lamiaceae EOs showed the highest antimicrobial efficiency against many microorganisms as Esche- richia coli, Staphylococcus aureus, Aspergillus fumigatus, Candida albicans...etc) $[3,4]$. These properties could be attributed to the main constituents of Lamiaceae essential oils such as carvacrol, thymol, p-cymene, 1,8-cineole, caryophyllene [5-7].

Food borne diseases have become one of the public health problems as microbial resistance against lots of antibiotics has increased worldwide. Foodborne pathogens are the causes of illness and death in developing countries [8]. The spectrum of foodborne pathogens includes a variety of enteric bacteria, aerobes and anaerobes, viral pathogens and parasites [9]. Salmonella, Escherichia coli, Shigella and Staphylococcus aureus are considered as most 
common foodborne pathogens [10]. With the renaissance of "back to nature", increasing concerns over food safety and a high demand for eco-friendly natural products has led to a growing interest in natural antimicrobial compounds.

Essential oils exhibit a wide range of antimicrobial activities, and different plant extracts for food preservation [11]. The chemical composition and properties of the essential oils of plants growing in Bani Matar area (Yemen) have never been investigated before. So, in this study, the chemical composition of essential oils isolated from Lavandula pubescens Decne, Marrubium vulgare $\mathrm{L}$. and Meriandra bengalensis (Roxb.) Benth and their antimicrobial activities have been assessed.

\section{Materials and Methods}

Plant material

Three medicinal Lamiaceae taxa were collected; Lavandula pubescens, Marrubium vulgare and Meriandra bengalensis, during the rainy season in 2-22/8/2015 from different locations in Bani Matar District, Sanaa governorate, Yemen (Table 1). The identification of the specimens was done by utilizing the available taxonomic and floristic literatures [8-11]. Voucher specimens have been deposited at the Herbarium of Faculty of Pharmay, Ain Shams University (PHG-LP-303, PHG-MV-304, PHG-MB-305) and a duplicate of each herbarium specimen was kept at the Herbarium of Biology Department, Faculty of science Sanaa University.

\begin{tabular}{|l|c|c|c|c|}
\hline No. & Medicinal plant & Local name & \% yield* & Date \\
\hline 1 & Lavandula pubescens Decne. ${ }^{\text {a) }}$ & Vaheia & 1.00 & $14 / 9 / 2015$ \\
\hline 2 & Marrubium vulgare L.) & Hei wjazp & 0.05 & $20 / 5 / 2016$ \\
\hline 3 & Meriandra bengalensis (Roxb.)Benth. ${ }^{\text {c) }}$ & diarū & 5.00 & $10 / 9 / 2015$ \\
\hline \multicolumn{2}{|c|}{ Plants were collected at altitude 2402-3353 m nearby following villages: ${ }^{\text {a) }}$ Kusher; ${ }^{\text {b) }}$ Al-saih } \\
(Jabal An-Nabi Shuayb); ${ }^{\text {c) Kusher. }}$ \\
*\% v/w: volume of oil in mL obtained from 100 g of fresh plant material. \\
\hline
\end{tabular}

Table 1: Data of collection and yield of essential oil obtained from Lavandula pubescens, Marrubium vulgare and Meriandra bengalensis.

Isolation of the essential oil

The fresh leaves and green branches of the three plants were chopped into small pieces. The essential oil was isolated from each part by hydro distillation for $5 \mathrm{~h}$ using a Clevenger-type all glass apparatus. Each oil was transferred to a screw-capped glass vial, dried $\left(\mathrm{Na}_{2} \mathrm{SO}_{4}\right)$ and stored at $4{ }^{\circ} \mathrm{C}$ in the dark until analysis.

\section{Analysis of essential oils by GC and GC-MS}

GC analysis was carried out using a GC HP 5890 Hewlett Packard equipped with FID and HP-5 fused silica capillary column $30 \mathrm{~m} \times$ $0.25 \mathrm{~mm}$ i.d., film thickness $0.25 \mu \mathrm{m}$, using a sample volume of 0.03 $\mu \mathrm{L}$. Oven temperature was programmed from $60{ }^{\circ} \mathrm{C}$ to $240{ }^{\circ} \mathrm{C}$ at $3 \stackrel{\circ}{\circ} \mathrm{C} / \mathrm{min}$; injector temperature $250^{\circ} \mathrm{C}$; detector temperature 280 ${ }^{\circ} \mathrm{C}$; carrier gas was helium, flow rate was $1.0 \mathrm{~mL} / \mathrm{min}$; automatic sample injection, $0.02 \mu \mathrm{L}$ of the oil; split was $1 / 70$. The relative proportions of the essential oil constituents were expressed as percentages obtained by peak area normalization. GC-MS analysis was performed on a Perkin-Elmer quadrupole MS system (Model 5) coupled with the GC HP 5972, equipped with a HP-5 capillary column. Oven temperature was programmed from $45{ }^{\circ} \mathrm{C}$ to $240{ }^{\circ} \mathrm{C}$ at $3{ }^{\circ} \mathrm{C} / \mathrm{min}$; injector temperature $250 \stackrel{\circ}{\circ}$; carrier gas was helium, flow rate was $0.5 \mathrm{~mL} / \mathrm{min}$; automatic sample injection, $0.02 \mu \mathrm{L}$ of the oil; split: 1/70. The MS operating parameters were interface temperature: $300{ }^{\circ} \mathrm{C}$, ion source temperature: $200{ }^{\circ} \mathrm{C}$, EI mode: 70 $\mathrm{eV}$, scan range was 41-400 amu.

\section{Identification of the components}

Mass spectra of the individual GC peaks were identified by a computer search of the commercial libraries (WILEY, NIST), as well as matching with published mass spectra [12-14]. The identification was further confirmed by the calculation of the retention indices (RI) relative to (C6-C22) $n$-alkanes [15].

\section{Antimicrobial screening}

A series of bacterial and fungal strains available in stock culture of the Regional Center for Mycology and Biotechnology Antimicrobial Unite (RCMB), Cairo, Egypt, were used for antibiotic sensitivity testing comprising: Gram-positive Bacteria [Staphylococcus aureus (RCMB 010067) Enterococcus faecalis (RCMB 010028); Bacillus subtilis (RCMB 010063)], Gram-negative Bacteria [Enterobacter cloacae (RCMB 010072); Klebsiella pneumoniae (RCMB 010052); Escherichia coli (RCMB 010093], and Fungi [Aspergillus fumigatus (RCMB 02568), Candida albicans (RCMB 05036), Saccharomyces cerevisiae (RCMB 05177);]. The previously prepared essential oil 
was diluted $1 / 3 \mathrm{v} / \mathrm{v}$ in dimethyl sulphoxide (E-Merck), $30 \mu \mathrm{l}$ (containing $10 \mu \mathrm{l}$ of pure oil) were used in the test. Dimethyl sulphoxide $(50 \mu \mathrm{l})$ was used as a negative control. The agar diffusion method [16] was applied using trypticase soy agar (Difco) medium inoculated with the bacterial or fungal suspension of the test organisms. Discs $5 \mathrm{~mm}$ in diameter were impregnated with the oils or the control. Then the discs were placed onto the surface of the culture medium. Discs of ampicillin, gentamycin and amphotericin B were used as standard antibacterial and antifungal agent, respectively. The plates were incubated at $35-37^{\circ} \mathrm{C}$ for $24-48$ hours in case of bacteria, $25^{\circ} \mathrm{C}$ for 48 hours in case of filamentous fungi, while yeasts were incubated at $30^{\circ} \mathrm{C}$ for $24-48$ hours. After incubation, the diameters of inhibition zones were recorded in millimeters and the results were compiled in table 4 . The minimum inhibitory concentrations (MIC) of S. sempervirens oil against the tested microorganisms were also determined by micro dilution method [17].

\section{Results and Discussion}

Many Lamiaceae plants induced antimicrobial activities against wide range of bacteria and fungi. For example, the antimicrobial properties of Dracocephalum kotschyi Boiss. were determined against 12 strains of microorganism where the highest activities were against Aspergillus brasiliensis, Bacillus subtilis and Candida albicans [18]. Meanwhile, the essential oils obtained from both leaves and flowers of Salvia hydrangea may have potential application as bactericidal agents against Pseudomonas aeruginosa, Shigella dysenteriae and Klebsiella pneumoniae [19].

Yield of oils and data of collection are listed in Table 1. The higher yield aws to Marrubium vulgare $5.00 \%$ v/w and the lowest one was $0.05 \% \mathrm{v} / \mathrm{w}$ for Meriandra bengalensis. Moderate yield was observed in Lavandula pubescens $(1.00 \% \mathrm{v} / \mathrm{w})$. Fourteen peaks were detected in Lavandula pubescens oil (90\%), (Table 2). Carvacrol represented the major peak (54.9\%), followed by thymol methyl ether (12.2\%). The most abundant constituents of the Marrubium vulgare chromatogram (88.9\%) were Z-caryophyllene (10.9\%), octadecanol (10.4\%), and $\alpha$-bisabolene (9.7\%), (Table 3). Seventeen peaks were recorded (99.6\%) in Meriandra bengalensis oil. The major constituents were camphor (64.9\%), 1,8-cineole (12.2\%) and camphene (12.0\%), (Table 4).

\begin{tabular}{|c|c|c|c|c|}
\hline No. & Formula & Compounds & KI & Oil Area [\%] ${ }^{\mathrm{a}}$ \\
\hline 1 & $\mathrm{C}_{10} \mathrm{H}_{16}$ & $\alpha$-Pinene & 936 & 0.23 \\
\hline 2 & $\mathrm{C}_{10} \mathrm{H}_{16}$ & $\beta$-Myrcene & 990 & 2.59 \\
\hline 3 & $\mathrm{C}_{10} \mathrm{H}_{16}$ & $\delta$-3 Carene & 1009 & 1.25 \\
\hline 4 & $\mathrm{C}_{10} \mathrm{H}_{14}$ & $p$-Cymene & 1028 & 0.79 \\
\hline 5 & $\mathrm{C}_{10} \mathrm{H}_{16}$ & Limonene & 1031 & 0.42 \\
\hline 6 & $\mathrm{C}_{10} \mathrm{H}_{16}$ & Terpinolene & 1087 & 4.87 \\
\hline 7 & $\mathrm{C}_{10} \mathrm{H}_{16} \mathrm{O}$ & trans-epoxy Ocimene & 1140 & 1.01 \\
\hline 8 & $\mathrm{C}_{10} \mathrm{H}_{12} \mathrm{O}_{2}$ & Phenyl ethyl acetate & 1194 & 1.64 \\
\hline 9 & $\mathrm{C}_{11} \mathrm{H}_{16} \mathrm{O}$ & Thymol methyl ether & 1231 & 12.15 \\
\hline 10 & $\mathrm{C}_{10} \mathrm{H}_{14} \mathrm{O}$ & Carvacrol & 1296 & 54.98 \\
\hline 11 & $\mathrm{C}_{15} \mathrm{H}_{24}$ & Z-Caryophyllene & 1404 & 4.47 \\
\hline 12 & $\mathrm{C}_{15} \mathrm{H}_{24}$ & $\alpha$-Bisabolene & 1507 & 1.30 \\
\hline 13 & $\mathrm{C}_{15} \mathrm{H}_{24} \mathrm{O}$ & Caryophyllene oxide & 1580 & 3.78 \\
\hline 14 & $\mathrm{C}_{20} \mathrm{H}_{32}$ & Cembrene & 1939 & 0.55 \\
\hline & & \multicolumn{2}{|c|}{ Total Identified } & 90.03 \\
\hline \multicolumn{2}{|c|}{ Functional group } & \multicolumn{3}{|c|}{ Total peak (\%) } \\
\hline \multicolumn{2}{|c|}{ Monoterpene hydrocarbons } & \multicolumn{3}{|c|}{10.15} \\
\hline \multicolumn{2}{|c|}{ Sesquiterpene hydrocarbons } & \multicolumn{3}{|c|}{5.77} \\
\hline \multicolumn{2}{|c|}{ Diterpene hydrocarbons } & \multicolumn{3}{|c|}{0.55} \\
\hline \multicolumn{2}{|c|}{ Oxygenated monoterpene } & \multicolumn{3}{|c|}{69.78} \\
\hline \multicolumn{2}{|c|}{ Oxygenated sesquiterpene } & \multicolumn{3}{|c|}{3.78} \\
\hline \multicolumn{2}{|c|}{ Total hydrocarbons compounds } & \multicolumn{3}{|c|}{16.47} \\
\hline \multicolumn{2}{|c|}{ Total oxygenated compounds } & \multicolumn{3}{|c|}{73.56} \\
\hline \multicolumn{5}{|c|}{$\begin{array}{c}\text { a Values are expressed as relative area percentage; }{ }^{b} \text { Kovats retention index calculated } \\
\text { on DB-5 column; The major components are highlighted in bold. (Values expressed as } \\
\text { relative area percentages to the total identified components). }\end{array}$} \\
\hline
\end{tabular}

Table 2: Essential oil composition of Lavandula pubescens. 


\begin{tabular}{|c|c|c|c|c|}
\hline No. & Formula & Compounds & $\mathbf{K I}$ & Oil Area $[\%]^{a}$ \\
\hline 1 & $\mathrm{C}_{10} \mathrm{H}_{16}$ & $\alpha$-Pinene & 936 & 2.27 \\
\hline 2 & $\mathrm{C}_{8} \mathrm{H}_{16} \mathrm{O}$ & Octadienal & 1105 & 2.23 \\
\hline 3 & $\mathrm{C}_{10} \mathrm{H}_{14} \mathrm{O}$ & Carvacrol & 1296 & 1.11 \\
\hline 4 & $\mathrm{C}_{15} \mathrm{H}_{24}$ & $\alpha$-Copaene & 1374 & 2.71 \\
\hline 5 & $\mathrm{C}_{15} \mathrm{H}_{24}$ & Z-Caryophyllene & 1404 & 10.95 \\
\hline 6 & $\mathrm{C}_{15} \mathrm{H}_{24}$ & $Z$ - $\beta$-Farnesane & 1444 & 6.91 \\
\hline 7 & $\mathrm{C}_{15} \mathrm{H}_{24}$ & trans Muuroladiene & 1457 & 3.97 \\
\hline 8 & $\mathrm{C}_{14} \mathrm{H}_{24} \mathrm{O}$ & $\alpha$-Ionol isomethyl & 1466 & 4.18 \\
\hline 9 & $\mathrm{C}_{15} \mathrm{H}_{24}$ & $\alpha$-Bisabolene & 1507 & 9.72 \\
\hline 10 & $\mathrm{C}_{15} \mathrm{H}_{24}$ & -Cadinene & 1515 & 4.35 \\
\hline 11 & $\mathrm{C}_{15} \mathrm{H}_{26} \mathrm{O}$ & Z-Nerolidol & 1534 & 1.06 \\
\hline 12 & $\mathrm{C}_{15} \mathrm{H}_{24} \mathrm{O}$ & Caryophyllene oxide & 1583 & 3.72 \\
\hline 13 & $\mathrm{C}_{14} \mathrm{H}_{24} \mathrm{O}_{3}$ & Isoborneol & 1632 & 1.76 \\
\hline 14 & $\mathrm{C}_{15} \mathrm{H}_{30} \mathrm{O}$ & Pentadecanone & 1697 & 1.46 \\
\hline 15 & $\mathrm{C}_{12} \mathrm{H}_{14} \mathrm{O}_{2}$ & $E$-Ligustilide & 1796 & 1.82 \\
\hline 16 & $\mathrm{C}_{20} \mathrm{H}_{40} \mathrm{O}$ & Phytol & 1943 & 2.54 \\
\hline 18 & $\mathrm{C}_{20} \mathrm{H}_{34} \mathrm{O}$ & Z, Z-Geranyl linalol & 1961 & 5.86 \\
\hline 19 & $\mathrm{C}_{20} \mathrm{H}_{36} \mathrm{O}$ & Pseudo phytol & 1988 & 1.95 \\
\hline 20 & $\mathrm{C}_{11} \mathrm{H}_{10} \mathrm{O}_{3}$ & $\begin{array}{c}\text { 7-Hydroxy-4,8-dimethyl Cou- } \\
\text { marin }\end{array}$ & 2013 & 1.35 \\
\hline 21 & $\mathrm{C}_{20} \mathrm{H}_{34} \mathrm{O}$ & Manool & 2060 & 1.98 \\
\hline 22 & $\mathrm{C}_{18} \mathrm{H}_{38} \mathrm{O}$ & Octadecanol & 2077 & 10.44 \\
\hline 23 & $\mathrm{C}_{18} \mathrm{H}_{34} \mathrm{O}_{2}$ & Oleic acid & 2142 & 1.21 \\
\hline 24 & $\mathrm{C}_{20} \mathrm{H}_{40} \mathrm{O}_{2}$ & Octadecenol acetate & 2209 & 5.36 \\
\hline & & Total Identified & & 88.91 \\
\hline & \multicolumn{2}{|c|}{ Functional group } & \multicolumn{2}{|c|}{ Total peak (\%) } \\
\hline & \multicolumn{2}{|c|}{ Monoterpene hydrocarbons } & \multicolumn{2}{|r|}{2.27} \\
\hline & \multicolumn{2}{|c|}{ Sesquiterpene hydrocarbons } & \multicolumn{2}{|c|}{38.61} \\
\hline & \multicolumn{2}{|c|}{ Oxygenated monoterpene } & \multicolumn{2}{|c|}{3.34} \\
\hline & \multicolumn{2}{|c|}{ Oxygenated sesquiterpene } & \multicolumn{2}{|r|}{14} \\
\hline & \multicolumn{2}{|c|}{ Oxygenated diterpene } & \multicolumn{2}{|c|}{30.69} \\
\hline & \multicolumn{2}{|c|}{ Total Hydrocarbons compound } & \multicolumn{2}{|c|}{40.88} \\
\hline & \multicolumn{2}{|c|}{ Total oxygenated compound } & \multicolumn{2}{|c|}{48.03} \\
\hline \multicolumn{5}{|c|}{ 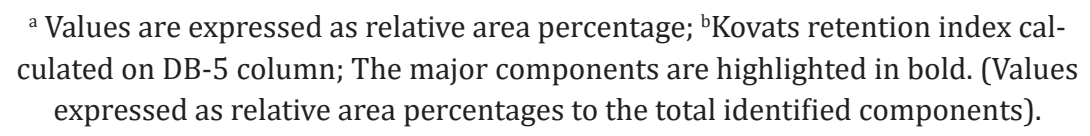 } \\
\hline
\end{tabular}

Table 3: Essential oil composition of Marrubium vulgare. 


\begin{tabular}{|c|c|c|c|c|}
\hline No. & Formula & Compounds & KI & Oil Area [\%] ${ }^{a)}$ \\
\hline 1 & $\mathrm{C}_{10} \mathrm{H}_{16}$ & Tricyclene & 924 & 0.57 \\
\hline 2 & $\mathrm{C}_{10} \mathrm{H}_{16}$ & $\alpha$-Pinene & 936 & 3.45 \\
\hline 3 & $\mathrm{C}_{10} \mathrm{H}_{16}$ & Camphene & 953 & 11.96 \\
\hline 4 & $\mathrm{C}_{10} \mathrm{H}_{16}$ & Sabinene & 975 & 0.22 \\
\hline 5 & $\mathrm{C}_{10} \mathrm{H}_{16}$ & $\beta$-Pinene & 981 & 1.24 \\
\hline 6 & $\mathrm{C}_{10} \mathrm{H}_{16}$ & $\delta$-3Carene & 1009 & 0.97 \\
\hline 7 & $\mathrm{C}_{10} \mathrm{H}_{18} \mathrm{O}$ & 1,8 cineole & 1035 & 12.16 \\
\hline 8 & $\mathrm{C}_{9} \mathrm{H}_{14} \mathrm{O}$ & Camphenilone & 1085 & 0.23 \\
\hline 9 & $\mathrm{C}_{10} \mathrm{H}_{16} \mathrm{O}$ & trans-Pinocarveol & 1143 & 0.36 \\
\hline 10 & $\mathrm{C}_{10} \mathrm{H}_{16} \mathrm{O}$ & Camphor & 1147 & 64.87 \\
\hline 11 & $\mathrm{C}_{10} \mathrm{H}_{18} \mathrm{O}$ & Borneol & 1175 & 1.33 \\
\hline 12 & $\mathrm{C} 10 \mathrm{H}_{16} \mathrm{O}$ & cis-Pinocarveol & 1185 & 0.16 \\
\hline 13 & $\mathrm{C}_{10} \mathrm{H}_{14} \mathrm{O}$ & Myrtenal & 1198 & 0.21 \\
\hline 14 & $\mathrm{C}_{10} \mathrm{H}_{14} \mathrm{O}$ & Verbenone & 1208 & 0.35 \\
\hline 15 & $\mathrm{C}_{15} \mathrm{H}_{24} \mathrm{O}$ & Caryophyllene Oxide & 1580 & 0.24 \\
\hline 16 & $\mathrm{C}_{15} \mathrm{H}_{26} \mathrm{O}$ & Eudesmol-epi- & 1620 & 0.16 \\
\hline 17 & $\mathrm{C}_{15} \mathrm{H}_{26} \mathrm{O}$ & Eudesmol & 1630 & 1.11 \\
\hline & & Total all & & 99.59 \\
\hline & ional group & & Tot & ak $(\%)$ \\
\hline \multicolumn{3}{|c|}{ Monoterpene hydrocarbons } & \multicolumn{2}{|c|}{18.41} \\
\hline \multicolumn{3}{|c|}{ Oxygenated monoterpene } & \multicolumn{2}{|c|}{79.67} \\
\hline \multicolumn{3}{|c|}{ Oxygenated sesquiterpene } & \multicolumn{2}{|c|}{1.51} \\
\hline \multicolumn{3}{|c|}{ Total hydrocarbon compounds } & \multicolumn{2}{|c|}{18.41} \\
\hline \multicolumn{3}{|c|}{ Total oxygenated compounds } & \multicolumn{2}{|c|}{81.18} \\
\hline \multicolumn{5}{|c|}{ 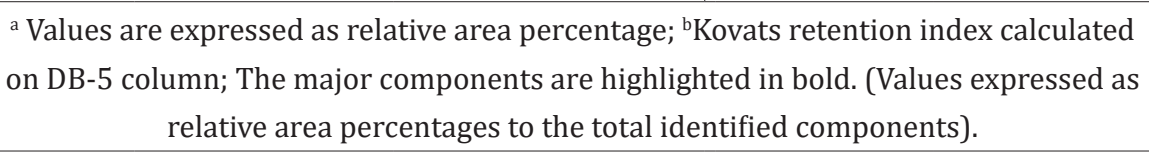 } \\
\hline
\end{tabular}

Table 4: Essential oil composition of Meriandra bengalensis.

Reported literatures concerning Lavandula pubescens oil revealed that carvacrol was the major compound in the oil (60.970\%) [20]. It is the major peak in the leaf oil of eight wild Lavandula pubescens plants from different locations in Yemen [20].

Different compositional patterns appear obviously upon comparing our data with previous studies regarding EO of Marrubium vulgare. Iranian oil contained $\beta$-caryophyllene (32.2\%), (E)- $\beta$-farnesene (11.4\%) and 1,8-cineole (8.2\%) [21]. Essential oil isolated from Egyptian species composed of carvacrol (36.3\%) and $\beta$-phellandrene (15.5\%) [22]. Turkeian oil composed of $\alpha$-pinene (28.9\%), $\beta$-pinene (18.3\%) and $\beta$-phellandrene (17.4\%) [23]. Carvacrol content is quite high which explains the antimicrobial effect on both Gram negative and Gram positive bacteria as well as fungi. The antimicrobial effect of carvacrol on bacterial cells has been widely explained [24,25].

The content of camphor (64.9\%) in our sample of Meriandra bengalensis EO was close with those previously reported in Yemen (43.6\%). The major component from Indian oil [26] was l-linalool 
(68.4\%) followed by 1,8-cineole (17.4\%) that agrees with our result. These differences in components might be attributed to climatic and environmental conditions [27].

Tables 5 and 6 displayed zone of inhibition and minimum inhibitory concentration (MIC) of the three oils. According to literatures which pointed to the potency of a drug according to its MIC: MIC of $<0.5 \mathrm{uL} / \mathrm{mL}$ are considered potent; 0.6-1.5 uL/mL are considered moderate; > $1.5 \mathrm{uL} / \mathrm{mL}$ are considered weak [28,29]. Lavandula pubescens oil induced potent antibacterial activities against Enterobacter cloacae (0.98 $\mu \mathrm{L} / \mathrm{mL})$, Klebsielle pneumoniae $(1.95 \mu \mathrm{L} / \mathrm{mL})$, which is higher than that of gentamycin and Bacillus subtilis $(0.49$ $\mu \mathrm{L} / \mathrm{mL}$ ) that is of similar to ampicillin activity. It showed moderate activity against Escherichia coli and Saccharomyces cerevisiae (1.95 and $0.98 \mu \mathrm{L} / \mathrm{mL}$ respectively). A weak activity was induced against Enterococcus faecalis and Aspergillus fumigatus (7.81 and $1.95 \mu \mathrm{L}$ / $\mathrm{mL}$ ). No activity was observed against Candida albicans.
Meriandra bengalensis exhibited moderate activitiy against Saccharomyces cerevisiae $(3.9 \mu \mathrm{L} / \mathrm{mL})$ and weak or even no activity against the rest of tested microorganisms.

Also, the EO of Lavandula pubescens induced weak activities against Escherichia coli, Staphylococcus aureus, Aspergillus fumigatus and Candida albicans, and no activity against the rest tested organisms.

This diversity of activity of the tested oils could be attributed mainly to the presence of carvacrol thymol methyl ether, Z-caryophyllene. It was reported that carvacrol induces antimicrobial activity [30] on some bacteria as E. coli and Salmonella typhimurium; (MIC: 1 and $3 \mathrm{mM}$ ) [31]. Thymol methyl ether was the main constituent of Satureja sp, and Thymus fallax oils that exhibited antimicrobial activity ( MIC values of the oils vary with the bacterial strain tested, ranging from 15.63 to

\begin{tabular}{|l|c|c|c|c|c|c|c|c|c|}
\hline \multirow{3}{*}{ Plant } & \multicolumn{9}{|c|}{ Diameter of inhibition zone (mm) ${ }^{\text {a)b)cd) }}$} \\
\cline { 2 - 10 } & \multicolumn{3}{|c|}{ Gram-negative bacteria } & \multicolumn{2}{c|}{ Gram-positive bacteria } & \multicolumn{4}{c|}{ Fungal species } \\
\cline { 2 - 10 } & EC & KP & Eco & SA & EF & BS & AF & CA & SC \\
\hline LP & $22.9 \pm 0.37$ & $21.4 \pm 0.58$ & $20.7 \pm 0.63$ & $22.3 \pm 0.63$ & $18.1 \pm 0.72$ & $24.2 \pm 0.25$ & $21.2 \pm 0.58$ & NA & $22.4 \pm 0.58$ \\
\hline MV & NA & $11.6 \pm 0.63$ & $16.3 \pm 0.63$ & NA & $15.2 \pm 1.2$ & NA & $12.3 \pm 1.2$ & $15.2 \pm 0.72$ & NA \\
\hline MB & $16.2 \pm 0.44$ & $15.8 \pm 0.12$ & $18.2 \pm 0.44$ & $16.2 \pm 0.37$ & NA & $18.3 \pm 0.37$ & $18.2 \pm 0.25$ & NA & $18.9 \pm 1.2$ \\
\hline \multirow{3}{*}{ Standard } & \multicolumn{3}{|c|}{ Gentamicin } & \multicolumn{3}{|c|}{ Ampicillin } & \multicolumn{3}{|c|}{ Amphotericin B } \\
\cline { 2 - 10 } & $23.8 \pm 0.63$ & $20.2 \pm 0.12$ & $27.3 \pm 0.44$ & $28.9 \pm 0.14$ & $25.3 \pm 0.58$ & $26.4 \pm 0.34$ & $23.7 \pm 0.10$ & $21.9 \pm 0.12$ & $27.8 \pm 0.58$ \\
\hline
\end{tabular}

Table 5: Antimicrobial activity of the volatile oils of Lavandula pubescens, Marrubium vulgare and Meriandra bengalensis as inhibition zone diameter.

a) Mean zone of inhibition in $\mathrm{mm}$ _ standard deviation beyond well diameter $(6 \mathrm{~mm})$ produced on a range of environmental and clinically pathogenic microorganisms. The concentration used for the standard antibiotic was $30 \mathrm{mg} / \mathrm{mL}$.

b) The test was done using the diffusion agar technique. Well diameter: $6.0 \mathrm{~mm}$. RCMB: Regional Center for Mycology and Biotechnology Antimicrobial unit test organisms.

c) Results are Mean \pm SD of triplicate values.

d) 6-9 mm: no activity; 12-15 mm: low activity; 15-18 mm: good activity; above $18 \mathrm{~mm}$ : significant activity.

LP: Lavandula pubescens Decne, MV: Marrubium vulgare L. and MB: Meriandra bengalensis

AF: Aspergillus fumigatus (RCMB 02568), CA: Candida albicans (RCMB 05036), SC: Saccharomyces cerevisiae (RCMB 05177); SA: Staphylococcus aureus (RCMB 010067); EF: Enterococcus faecalis (RCMB 010028); BS: Bacillus subtilis (RCMB 010063); EC: Enterobacter cloacae (RCMB 010072); KP: Klebsiella pneumoniae (RCMB 010052); ECo: Escherichia coli (RCMB 010093)

NA: no activity. 


\begin{tabular}{|c|c|c|c|c|c|c|c|c|c|}
\hline \multirow{2}{*}{ Plant } & \multicolumn{3}{|c|}{ Gram-negative bacteria } & \multicolumn{3}{|c|}{ Gram-positive bacteria } & \multirow[b]{2}{*}{$\mathbf{A F}$} & \multirow[b]{2}{*}{ CA } & \multirow[b]{2}{*}{ SC } \\
\hline & EC & KP & ECo & SA & EF & BS & & & \\
\hline LP & 0.98 & 1.95 & 1.95 & 0.98 & 7.81 & 0.49 & 1.95 & NA & 0.98 \\
\hline MV & NA & NA & 125 & 31.25 & NA & NA & 125 & 31.25 & NA \\
\hline MB & 31.25 & 31.25 & 7.81 & 15.63 & NA & 7.81 & 7.81 & NA & 3.9 \\
\hline \multirow[t]{2}{*}{ Standard } & \multicolumn{3}{|c|}{ Gentamicin } & \multicolumn{3}{|c|}{ Ampicillin } & \multicolumn{3}{|c|}{ Amphotericin B } \\
\hline & 0.98 & 3.9 & 0.98 & 0.49 & 0.98 & 0.49 & 0.49 & 0.98 & 0.49 \\
\hline
\end{tabular}

Table 6: Antimicrobial activities (MIC, $\mathrm{uL} / \mathrm{mL}$ ) of the volatile oils of Lavandula pubescens, Marrubium vulgare and Meriandra bengalensis

a) Mean zone of inhibition in $\mathrm{mm}$ _ standard deviation beyond well diameter ( $6 \mathrm{~mm}$ ) produced on a range of environmental and clinically pathogenic microorganisms. The concentration used for the standard antibiotic was $30 \mathrm{mg} / \mathrm{mL}$.

b) The test was done using the diffusion agar technique. Well diameter: $6.0 \mathrm{~mm}$. RCMB: Regional Center for Mycology and Biotechnology Antimicrobial unit test organisms.

c) Results are Mean \pm SD of triplicate values.

d) 6-9 mm: no activity; 12-15 mm: low activity; 15-18 mm: good activity; above $18 \mathrm{~mm}$ : significant activity.

LP: Lavandula pubescens Decne, MV: Marrubium vulgare L. and MB: Meriandra bengalensis

AF: Aspergillus fumigatus (RCMB 02568), CA: Candida albicans (RCMB 05036), SC: Saccharomyces cerevisiae (RCMB 05177); SA: Staphylococcus aureus (RCMB 010067); EF: Enterococcus faecalis (RCMB 010028); BS: Bacillus subtilis (RCMB 010063); EC: Enterobacter cloacae (RCMB 010072); KP: Klebsiella pneumoniae (RCMB 010052); ECo: Escherichia coli (RCMB 010093)

NA: no activity.

125.0 $\mu \mathrm{LmL}-1$ ) [32]. One of the major components identified in Nectandra megapotamica oil is Z-caryophyllene which exhibited strong activity against Streptococcus mutans (MIC: $50 \mu \mathrm{g} / \mathrm{mL}$ ), Streptococcus sobrinus (MIC: $20 \mu \mathrm{g} / \mathrm{mL}$ ), Prevotella nigrescens (MIC: $50 \mu \mathrm{g} / \mathrm{mL}$ ) and Bacteroides fragilis (MIC: $31.25 \mu / \mathrm{mL}$ ) [33]. 6-Caryophyllene demonstrated selective antibacterial activity against $S$. aureus (MIC $3 \pm 1.0 \mu \mathrm{M}$ ) and more pronounced antifungal activity than kanamycin [34]. Some researchers have demonstrated that whole EOs usually induce higher antimicrobial activity than the mixtures of their major compounds, suggesting that the minor compounds are critical to the synergistic activity, though antagonistic and additive effects have also been observed.

\section{Conclusions}

It could be concluded that the oil of Lavandula pubescens, is more effective as antimicrobial agent than Marrubium vulgare and Meriandra bengalensis. It showed strong antimicrobial activities against most tested pathogenic microbial and could be considered as potential natural antibiotic for many infections. Its essential oils showed a great importance to be clinically used in the treatment of various Klebsielle pneumoniae, Enterobacter cloacae and Bacillis subtilis Klebsielle pneumoniae, Enterobacter cloacae and Bacillis subtilis infections. However, its activity should be more deeply investigated before proposing it as a natural antibiotic for many infections. Moreover, its toxicity should be tested before any potential application on animal and human beings.

\section{Bibliography}

1. Cantino PD., et al. "A comparison of phylogenetic nomenclature with the current system: a botanical case study". System Biology 46 (1997): 313-331.

2. Kadereit J and Kubitzki K. "The Families and Genera of Vascular Plants VII. Flowering Plants Dicotyledons: Lamiales (Except Acanthaceae Including Avicenniaceae), Flowering Plants • Dicotyledons". Springer Berlin Heidelberg, Berlin, Heidelberg (2004).

3. Al-Khyat SH., et al. "Antimicrobial activity of Lavandula pubescens essential oil from two places In Yemen". Journal of Advances in Biology 4 (2014): 446-454. 
4. Chorianopoulos NG., et al. "Seasonal variation in the chemical composition of the essential oils of Satureja species and their MIC assays against foodborne pathogens". Food Chemistry 54 (2006a): 3139-3145.

5. Gayathiri K., et al. "Potential pharmacological uses of natural products from Lamiaceae". International Journal of Pharmaceutical Sciences Review and Research 5 (2016): 21-34.

6. Chorianopoulos NG., et al. "Essential oils of Lamiaceae family taxa as natural preservatives of food preparations". Food Chemistry 1.2 (2007): 202-215.

7. Nezhadali A., et al. "Chemical variation of leaf essential oil at different stages of plant growth and in vitro antibacterial activity of Thymus vulgaris Lamiaceae from Iran". Journal of Basic and Applied Sciences 3 (2014): 87-92.

8. Tassew H., et al. "Microbial flora and food borne pathogens on minced meat and their susceptibility to antimicrobial agents". Ethiopian Journal of Health Sciences 20 (2010): 137-143.

9. Vasickova P., et al. "Viruses as a cause of foodborne diseases: a review of the literature". Veterinary Medicine Czech 50 (2005): 89-104.

10. Pierce SE., et al. "Detection and identification of Salmonella enterica, Escherichia coli, and Shigella spp. via PCR-electrospray ionization mass spectrometry: isolate testing and analysis of food samples". Applied and Environmental Microbiology 78 (2012): 8403-8411.

11. Burt S. "Essential oils: their antibacterial properties and potential applications in foods--a review". International Journal of Food Microbiology 94 (2004): 223-253.

12. Azab SS., et al. "Anti-inflammatory and gastroprotective potential of leaf essential oil of Cinnamomum glanduliferum in ethanol-induced rat experimental gastritis". Pharmaceutical Biology 55 (2017): 1654-1661.

13. Eldahshan OA and Halim FA. "Comparison of the composition and antimicrobial activities of the essential oils of green branches and leaves of Egyptian Navel orange (Citrus sinensis (L.) osbeck var. malesy)". Chemistry and Biodiversity 13 (2016): 1-5.

14. Taha AS and Eldahshan OA. "Chemical characteristics, antimicrobial and cytotoxic activities of the essential oil of Egyptian Cinnamomum glanduliferum Bark". Chemistry and Biodiversity 11 (2017): e1600443e1600443.
15. Adams RP. "Identification of essential oil components by gas chromatography/mass spectrometry". Allured Publishing Corporation, Illinois, USA, 4th Edition (2007).

16. Bauer AW., et al. "Antibiotic susceptibility testing by a standardized single disk method". American Journal of Clinical Pathology 45 (1996): 493-496.

17. Farraro MJ., et al. "Performance Standards for Antimicrobial Disk Susceptibility Tests Approved Standard M2-A7". 7th Edn, National Committee for Clinical Laboratory Standards: Wayne, PA, USA (2000): 1-26.

18. Ghavam M., et al. "Extraction of essential oil from Dracocephalum kotschyi Boiss. (Lamiaceae), identification of two active compounds and evaluation of the antimicrobial properties". Journal of Ethnopharmacology 267 (2021): 113513.

19. Ghavam M., et al. "Chemical composition and antimicrobial activity of essential oils obtained from leaves and flowers of Salvia hydrangea DC. ex Benth". Scientific Report 10 (2020): 15647.

20. Al-Badani RN., et al. "Variations in essential oil compositions of Lavandula pubescens (Lamiaceae) aerial parts growing wild in Yemen". Chemistry and Biodiversity 14.3 (2017): 3.

21. Bayir B., et al. "Chemical composition of essential oil from Marrubium vulgare L. Leaves”. 6 (2014): 44-50.

22. Bokaeian M., et al. "Phytochemical analysis antibacterial activity of Marrubium vulgare L against Staphylococcus aureus in vitro". Zahedan Journal of Research in Medical Sciences 16 (2014): 60-64.

23. Said-Al Ahl HA., et al. "Essential oil composition of Marrubium vulgare L. cultivated in Egypt". International Journal of Plant Science and Ecology 1 (2015): 138-141.

24. Ultee A., et al. "The phenolic hydroxyl group of carvacrol is essential for action against the food-borne pathogen Bacillus cereus". Applied and Environmental Microbiology (2002): 1561-1568.

25. Xu J., et al. "The antibacterial mechanism of carvacrol and thymol against Escherichia coli". Letter of Applied of Microbiology 47.3 (2008): 174-179.

26. Rana VS and Basques MA. "Constituents of the essential oil of Meriandra bengalensis Benth. leaves from India". Journal of Essential Oil Research 21 (2009): 22-23. 
27. Golparvar R., et al. "Essential oil composition of Marrubium vulgare L. from Iran”. Journal of Herbal Drugs 6 (2015): 1-5.

28. Sartoratto A., et al. "Composition and antimicrobial activity of essential oils from aromatic plants used in Brazil". Brazilian Journal of Microbiology 35 (2004): 275-280.

29. Duarte MC., et al. "Activity of essential oils from Brazilian medicinal plants on Escherichia coli". Journal of Ethnopharmacology 4 (2007): 197-201.

30. Kachur K and Suntres Z. "The antibacterial properties of phenolic isomers, carvacrol and thymol". Critical Reviews in Food Science and Nutrition 16 (2019): 1-12.

31. Chimnoi N., et al. "Characterization of essential oil from Ocimum gratissimum leaves: Antibacterial and mode of action against selected gastroenteritis pathogens". Microbe Pathogen 118 (2018): 290-300.

32. Kotan R., et al. "Antibacterial activities of essential oils and extracts of Turkish Achillea, Satureja and Thymus species against plant pathogenic bacteria". Journal of the Science of Food and Agriculture 15 (2010): 145-160.

33. Almeida KCR., et al. "Biological properties and chemical composition of essential oil from Nectandra megapotamica (Spreng.) Mez. leaves (Lauraceae)". Natural Product Research 14 (2019): 1-5.

34. Dahham SS., et al. "The anticancer, antioxidant and antimicrobial properties of the Sesquiterpene $\beta$-Caryophyllene from the Essential Oil of Aquilaria crassna". Molecules 20.7 (2015): 11808-11829.

\section{Volume 5 Issue 10 October 2021}

\section{(C) All rights are reserved by Eldahshan OA., et al.}

